\title{
Populações de aranhas errantes do gênero Ctenus em fragmentos florestais na Amazônia Central
}

\author{
Luiz Augusto Macedo MESTRE ${ }^{1}$, Thierry Ray GASNIER ${ }^{2}$
}

\begin{abstract}
RESUMO
Ctenus é um gênero de aranhas errantes abundante em florestas tropicais da América do Sul e da África e está se tornando um organismo modelo para compreensão da biologia de predadores na fauna de serapilheira. Avaliamos os efeitos da fragmentação florestal sobre quatro espécies de aranhas errantes do gênero Ctenus (C. amphora, C. crulsi, C. manauara e C. villasboasi), baseado nas variações das densidades e nos tamanhos de adultos em fragmentos de tamanhos diferentes, nas bordas destes e nas florestas secundárias próximas. Este estudo foi realizado de fevereiro a julho de 1999, em floresta tropical úmida de terra firme ao norte de Manaus - AM. As áreas incluíram três locais de mata secundária, quatro fragmentos de 1 ha, três de 10 ha, dois de 100 ha e quatro reservas em floresta contínua (maior que $10.000 \mathrm{ha}$ ). As aranhas foram capturadas manualmente, mensuradas e contadas em transectos de $250 \times 5 \mathrm{~m}$ ou 500x $5 \mathrm{~m}$ em áreas de platô, no interior e nas bordas dos fragmentos. Houve predomínio significativo de fêmeas em duas espécies (C. amphora-74\% e C. crulsi-65\%); dimorfismo sexual de tamanho em três espécies (C. amphora, C. crulsi e C. manauara) e variação temporal da abundância significativa para as quatro espécies. Estes resultados corroboraram e complementaram tendências de estudos anteriores e permitem uma interpretação mais completa sobre a ecologia destas espécies. Não observamos diferenças de abundância das aranhas entre centro e bordas e entre reservas de diferentes tamanhos. O efeito de borda ou do tamanho do fragmento sobre o tamanho de aranhas Ctenus neste estudo foi bem menor que as variaçôes anteriormente observadas entre habitats diferentes no interior da floresta. Comparaçôes com um estudo posterior a este, mostram que efeito da fragmentação nesta escala sobre estas aranhas diminui com a regeneração da floresta ao redor dos fragmentos, indicando uma rápida resposta do grupo para estas modificaçóes ambientais.
\end{abstract}

PALAVRAS-CHAVE: Aranhas errantes, Ctenus, Populaçôes, Amazônia, Fragmentos Florestais.

\section{Populations of Ctenus wandering spiders in Amazonian forest fragments}

\begin{abstract}
Ctenus is a genus of wandering spiders abundant in Neotropical and African rainforests and is becoming a model organism to understand the biology of predators in the leaf litter fauna. We compared abundance, sex ratio, seasonality and size dimorphism in populations of four species of medium sized wandering spiders, Ctenus amphora, C. crulsi, C. manauara and C. villasboasi in primary forests, fragments with different areas, their borders and secondary forests near them. This study was conducted between February and July 1999, in a terra-firme (non-flooded) tropical rain forest, in central Amazonia. The spiders were measured and counted in transects of $250 \times 5 \mathrm{~m}$ or 500x5 m in three secondary forest sites, four fragments of primary forest of $1 \mathrm{ha}$, three of $10 \mathrm{ha}$, two of 100 ha and four reserves of continuous forest (larger than $10.000 \mathrm{ha}$ ). There was a significant predominance of females in two species (C. amphora- 74\% and C. crulsi- 65\%), sexual dimorphism significant in three species (C. amphora, C. crulsi, and C. manauara), and temporal variation of the abundance for the four species. These results corroborate and complement tendencies from previous studies. There were no statistical differences between spider abundance in interior, borders and between reserves of different areas. Effects of border and size of fragments on the Ctenus spider populations are smaller than the previously observed variations among different habitats in the forest interior. There was no difference in spider size captured in small reserves, large reserves, edges, and continuous forests sites. Comparing these results with other study in the same sites, we conclude that the effects of fragmentation in Ctenus spiders decrease with second grown regeneration, resulting in a rapid reply of these species to habitat modifications.
\end{abstract}

KEYWORDS: Wandering spiders, Ctenus, Populations, Amazonia, Forest Fragments.

1 Projeto Dinâmica Biológica de Fragmentos Florestais, Instituto Nacional de Pesquisas da Amazônia, Smithsonian Tropical Research Institute, CP 478, 69083-000, Manaus - AM, Brasil. e-mail: Imestre@hotmail.com

2 Universidade Federal do Amazonas - Depto de Biologia / ICB, Av. Gal. Rodrigo Otávio Jordão Ramos 3000, CEP 69067-000, Manaus - Amazonas - Brasil. e-mail: tgasnier@ufam.edu.br 


\section{INTRODUÇÃO}

A fragmentação de habitats afeta a abundância e a riqueza de comunidades florestais, porém, estas respostas diferem entre os variados grupos biológicos (e.g. Lyntch \& Whitcomb, 1978; Harris, 1988; Bierregaard, et al. 1992; Hagan, 1996). Os efeitos do isolamento e borda já foram estudados em alguns grupos de insetos, como borboletas (Baz \& Garcia-Boyero, 1995; Brown \& Hutchings, 1997), abelhas (Lovejoy, 1980; Lovejoy et al., 1983; Lovejoy et al., 1986), besouros (Klein, 1989, Didham et al., 1996; Didham et al., 1998), cupins (Souza \& Brown, 1994) e formigas (Offerman et al., 1995, Vasconcelos et al., 2001). Porém, poucos estudos enfocaram outros artrópodos, principalmente grupos dependentes da disponibilidade de presas, como as aranhas.

Os poucos estudos que enfocaram aranhas em fragmentos florestais mostraram que as populaçōes são distintamente afetadas, pois a fragmentação pode favorecer mudanças na abundância e riqueza de espécies e até promover extinçôes locais (Miyashita et al., 1998; Bolger et al., 2000; Venticinque $\&$ Fowler, 2001). As comunidades de aranhas construtoras de teias são menos ricas em fragmentos urbanos no Japão, sendo que as aranhas de maior porte da família Araneidae parecem ser mais sensíveis à fragmentação (Miyashita et al., 1998). Por outro lado, Baldissera et al. (2004) detectaram o aumento da riqueza e abundancia de aranhas de teia na borda de áreas de florestas com Araucária. Estudos também mostraram que o tamanho de algumas aranhas pode refletir a qualidade do ambiente (Jocqué, 1981a, 1981b, Miyashita et al., 1998). Assim, é possível supor que as diferentes estratégias de captura de presas e/ou necessidades ecológicas das aranhas errantes podem estar favorecendo diferentes respostas a estas perturbações. No entanto, ainda são escassas as informações sobre quais são as respostas deste grupo ao impacto causado pela fragmentação de florestas.

Aranhas errantes da família Ctenidae, e o gênero Ctenus em particular, devido à sua abundância, têm recebido crescente interesse como bioindicadores de qualidade ambiental e como modelos para estudos de fatores que afetam a abundância de invertebrados predadores em florestas tropicais na África e na América do Sul (Gasnier, 1996; Almeida et al., 2000; Gasnier \& Höfer, 2001; Salvestrini \& Gasnier, 2001; FollyRamos et al., 2002; Gasnier et al., 2002; Jocqué et al., 2005; Rego et al., 2005, Gasnier et al., no prelo). Rego et al. (2005) encontraram evidências que a fragmentação florestal reduz as populações de aranhas do gênero Ctenus na Amazônia Central. As espécies Ctenus amphora (Mello-Leitão, 1930), C. crulsi (Mello-Leitão, 1930), C. manauara (Höfer, et al., 1994) e C. villasboasi (Mello-Leitão, 1949) coexistem na serapilheira do chão na floresta de terra firme da Amazônia Central, mas suas abundâncias absolutas e relativas variam bastante dentro e entre diferentes habitats da floresta (Gasnier
\& Höfer, 2001), portanto, o efeito da fragmentação poderia diferir entre as espécies em função de diferenças ecológicas que existem entre elas.

O objetivo deste estudo foi avaliar o efeito de fragmentação florestal sobre as populações destas quatro espécies de aranhas Ctenus, baseado nas variações das densidades e nos tamanhos de adultos, no centro e nas bordas de fragmentos de tamanhos diferentes, nas florestas secundárias e nas florestas primárias. Além disto, foram levantados dados que aumentam o conhecimento sobre a ecologia o gênero.

\section{MATERIAL E MÉTODOS}

Este estudo foi realizado em reservas florestais administradas pelo Projeto Dinâmica Biológica de Fragmentos Florestais do convênio INPA/Smithsonian Institution, caracterizadas como floresta tropical úmida de terra-firme, situadas ao norte de Manaus. A temperatura na região varia pouco $\left(25.8^{\circ} \mathrm{C}\right.$ a $27.9^{\circ}$ C) (Salati et al., 1991). Os meses mais chuvosos são de janeiro a maio e os mais secos são de julho a novembro, com média anual de chuvas de $2480 \mathrm{~mm}$ (Marques-Filho et al., 1981).

As áreas de estudo incluíram três locais de mata secundária maiores que $10 \mathrm{ha}(\mathrm{Sec})$, quatro fragmentos de $1 \mathrm{ha}(1 \mathrm{C}, 1 \mathrm{P}$, $1 \mathrm{D}, 1 \mathrm{E})$, três de 10 ha $(10 \mathrm{C}, 10 \mathrm{P}, 10 \mathrm{D})$, dois de 100 ha (100P, 100D), localizados na fazenda Colosso (1C e 10C), na fazenda Porto Alegre (1P, 10P e 100P) e na fazenda Dimona (1D, 1E, $10 \mathrm{D}$ e $100 \mathrm{D})\left(2^{\circ} 20^{\prime} 00^{\prime \prime} \mathrm{S} / 60^{\circ} 00^{\prime} 00^{\prime \prime} \mathrm{W}\right)$, também foram amostrados quatro locais em reservas de florestas contínuas, sendo um na reserva Adolfo Ducke $(\mathrm{CDu})$, um na reserva do $\mathrm{Km}$ 41(C41), um na reserva do Florestal (CFl) e um na floresta contínua na fazenda Dimona (CDi). Para descrições mais detalhadas destas áreas ver Lovejoy (1980), Lovejoy et al. (1983) e Stoufer \& Bierregaard (1995).

As amostragens foram realizadas de fevereiro a julho de 1999, apenas em noites sem luar ou chuva (entre 19:00 e 04:00 h). Foram feitas três excursōes para cada área amostral (com exceção de duas matas secundárias onde apenas foi possível realizar duas excursōes). As amostragens consistiram em percorrer (com velocidade constante aproximada de 500 $\mathrm{m} / \mathrm{h}$ ) transectos no interior e nas bordas dos fragmentos utilizando-se método de inspeção visual com auxílio de lanternas de cabeça. Os transectos eram estabelecidos aleatoriamente nas trilhas das reservas, sendo seu comprimento variável com a extensão da trilha disponível no local. Estes transectos possuíam 250 ou $500 \mathrm{~m}$ de comprimento por cinco metros de largura, não eram necessariamente contínuos ou retilíneos e localizavam-se sempre em áreas de platô (ambientes de latossolo não hidromórfico). Os indivíduos eram capturados manualmente, identificados, determinados o sexo, mensurados sempre com a mesma régua (com precisão de $1 \mathrm{~mm}$ ) o comprimento do prossoma (entre o fim do abdome 
e o início do cephalotórax excluindo as quelíceras) e soltos. A identificação em loco foi baseada na disposição dos olhos, dos desenhos no abdome e nas características visíveis (em lupa manual) das estruturas reprodutivas. Em caso de dúvida na identificação, os exemplares eram coletados e identificados em laboratório sob lupa. Alguns indivíduos foram depositados na coleção entomológica do INPA (números de tombo: 67644 a 67659).

As análises estatísticas foram baseadas em números de indivíduos em transectos de 500x $5 \mathrm{~m}$ (freqüência de indivíduos em $2500 \mathrm{~m} 2$ ). Foram agrupadas as reservas pequenas (1 ha a e 10 ha), reservas grandes (100 ha e Contínuas) e bordas. Foi utilizada estatística não paramétrica Wilcoxon-Sign-Rank-Test (WSRT); Kruskal-Wallis (KW) e Mann-Whitney (MW) nas comparações entre densidade e entre tamanhos dos indivíduos de cada espécie. Para reduzir a variação devida ao dimorfismo de tamanho, optou-se por utilizar apenas o tamanho das fêmeas nas avaliações de variação espacial do tamanho. A falta de duas amostragens nas áreas secundárias implicou na não inclusão destas nas análises estatísticas.

\section{RESULTADOS}

Observamos 513 indivíduos do gênero Ctenus, sendo 99 C. amphora, 227 C. crulsi, 150 C. manauara e 37 C. villasboasi. A proporção de fêmeas em relação a machos foi $74.4 \%$ para C. amphora, $64.5 \%$ para C. crulsi, $50.3 \%$ para C. manauara e $64.5 \%$ para C. villasboasi. Nas primeiras duas espécies, a proporção de fêmeas foi estatisticamente maior que $50 \%\left(X^{2}\right.$ $=27,2$ e 22,6 respectivamente, $\mathrm{P}<0.001)$.

A densidade das quatro espécies de Ctenus na primeira amostragem (entre fevereiro e abril) foi significativamente menor que na terceira amostragem (entre maio e julho) (Tabela 1). Esta redução foi estatisticamente significativa tanto para as quarto espécies juntas, quanto para C. crulsi, C. manauara e C. villasboasi separadamente (Wilcoxon Sign Rank Test Z= $-2.50,-3.08$ e -2.14 respectivamente, $\mathrm{P}<0.05$ para todas as espécies).

Não houve diferença estatisticamente significativa na densidade de aranhas entre reservas pequenas, grandes e bordas $(\mathrm{KW}=1.62,0.98,3.45,3.45$, para $C$. amphora, $C$.

Tabela 1 - Densidade (ind. $\mathrm{m}^{-2}$ ) de cada uma das quatro espécies de Ctenus nas amostragens em ordem cronológica ( 1 = fevereiro-abril, 2 = março-maio, $3=$ maio-julho). ( $b=$ Borda, $c=$ Centro). Os códigos das reservas estão no texto.

\begin{tabular}{|c|c|c|c|c|c|c|c|c|c|c|c|c|}
\hline \multirow[b]{2}{*}{ Reservas } & \multicolumn{3}{|c|}{ C.amphora } & \multicolumn{3}{|l|}{ C.crulsi } & \multicolumn{3}{|c|}{ C.manauara } & \multicolumn{3}{|c|}{ C.villasboasi } \\
\hline & 1 & 2 & 3 & 1 & 2 & 3 & 1 & 2 & 3 & 1 & 2 & 3 \\
\hline $1 \mathrm{Cb}$ & 0 & 0 & 0.0008 & 0.0008 & 0.0004 & 0 & 0 & 0.002 & 0 & 0 & 0 & 0 \\
\hline $1 \mathrm{Cc}$ & 0 & 0 & 0 & 0 & 0.0024 & 0.0008 & 0.0008 & 0.0016 & 0.0008 & 0 & 0 & 0 \\
\hline $1 \mathrm{~Pb}$ & 0 & 0.0016 & 0 & 0.0032 & 0.0016 & 0.004 & 0.0008 & 0 & 0 & 0 & 0 & 0 \\
\hline $1 \mathrm{Pc}$ & 0 & 0 & 0.0008 & 0.0024 & 0.0016 & 0.0032 & 0.0048 & 0.0024 & 0.0024 & 0.0008 & 0 & 0 \\
\hline $1 \mathrm{Db}$ & 0.0016 & 0 & 0 & 0.0008 & 0.0024 & 0.0008 & 0.0008 & 0.0016 & 0.0008 & 0 & 0 & 0 \\
\hline 1Dc & 0 & 0.0016 & 0 & 0.004 & 0.0008 & 0.0016 & 0.0032 & 0.004 & 0.0008 & 0 & 0.0008 & 0 \\
\hline $1 \mathrm{~Eb}$ & 0.0032 & 0.0008 & 0 & 0.0024 & 0.0024 & 0.0008 & 0 & 0 & 0 & 0.0016 & 0 & 0 \\
\hline $1 \mathrm{Ec}$ & 0 & 0.0008 & 0.0032 & 0.0016 & 0 & 0.0016 & 0.0008 & 0 & 0 & 0 & 0 & 0 \\
\hline $10 \mathrm{Cb}$ & 0.002 & 0 & 0.0004 & 0.0016 & 0.0028 & 0 & 0.0012 & 0 & 0.0008 & 0.0004 & 0.0004 & 0 \\
\hline $10 \mathrm{Cc}$ & 0 & 0.0012 & 0 & 0.0008 & 0.0024 & 0 & 0.0008 & 0.0008 & 0.0012 & 0 & 0 & 0 \\
\hline $10 \mathrm{~Pb}$ & 0.0008 & 0.0004 & 0.0016 & 0.0024 & 0.0028 & 0.0016 & 0.0008 & 0 & 0 & 0.0008 & 0 & 0.0008 \\
\hline $10 \mathrm{Pc}$ & 0.0008 & 0 & 0.0004 & 0.0036 & 0.0016 & 0.0008 & 0 & 0.0004 & 0.0004 & 0.0008 & 0 & 0 \\
\hline $10 \mathrm{Db}$ & 0.0008 & 0.0004 & 0.0016 & 0.0028 & 0.0012 & 0.0008 & 0.0032 & 0 & 0.0008 & 0.0008 & 0 & 0 \\
\hline $10 \mathrm{Dc}$ & 0.0004 & 0 & 0 & 0.002 & 0.0008 & 0.0004 & 0.0028 & 0 & 0.0012 & 0.0008 & 0 & 0 \\
\hline $100 \mathrm{~Pb}$ & 0.0004 & 0.0008 & 0 & 0.0008 & 0.0032 & 0 & 0 & 0.0004 & 0 & 0 & 0 & 0 \\
\hline $100 \mathrm{Pc}$ & 0.0004 & 0.002 & 0 & 0.0024 & 0.0016 & 0.0012 & 0.0004 & 0.0004 & 0.0008 & 0 & 0.0008 & 0.0004 \\
\hline $100 \mathrm{Db}$ & 0.0008 & 0.002 & 0.0004 & 0.004 & 0.0012 & 0.0012 & 0.0008 & 0.0008 & 0.0012 & 0.0008 & 0 & 0 \\
\hline $100 \mathrm{Dc}$ & 0.0032 & 0 & 0 & 0.0028 & 0.0016 & 0 & 0.0032 & 0.0008 & 0.0024 & 0 & 0.0004 & 0 \\
\hline C41c & 0.0024 & 0 & 0.0008 & 0.0028 & 0.0012 & 0.0004 & 0.0064 & 0.0004 & 0 & 0 & 0 & 0.0004 \\
\hline CDuc & 0.0008 & 0.0004 & 0.0004 & 0.002 & 0.0016 & 0.0008 & 0.0016 & 0.0028 & 0.0004 & 0.0004 & 0 & 0.0004 \\
\hline CDib & 0 & 0.0016 & 0 & 0 & 0.0016 & 0.0016 & 0.0008 & 0.0024 & 0 & 0.0008 & 0.0008 & 0.0008 \\
\hline CDib & 0 & 0 & 0 & 0 & 0 & 0.0008 & 0.0016 & 0 & 0 & 0.0008 & 0 & 0 \\
\hline CFlb & 0 & 0 & 0 & 0.0008 & 0.0008 & 0.0004 & 0.0012 & 0.0008 & 0.0012 & 0 & 0 & 0 \\
\hline CFlc & 0 & 0 & 0 & 0.0004 & 0 & 0.0004 & 0.002 & 0.0004 & 0.0008 & 0 & 0 & 0 \\
\hline SecC & 0.0004 & 0.002 & & 0.0016 & 0.0012 & & 0 & 0 & & 0 & 0 & \\
\hline SecP & 0 & 0.0012 & & 0.0004 & 0.0008 & & 0 & 0 & & 0.0016 & 0.0016 & \\
\hline SecD & 0.004 & 0.0016 & 0.0024 & 0.0024 & 0.0032 & 0.0048 & 0.0008 & 0 & 0 & 0.0008 & 0 & 0.0016 \\
\hline
\end{tabular}


crulsi, C. manauara e C. villasboasi, todas com $\mathrm{P}>0.05)$. A densidade de cada uma das espécies foi semelhante entre o centro das reservas de diferentes tamanhos e as bordas (figura 1). Apesar das áreas secundárias não estarem incluídas na avaliação estatística, é possível detectar um aparente aumento na proporção de $C$. amphora e $C$. villasboasi, e uma redução em C. manauara neste locais.

O tamanho das aranhas diferiu em função do sexo em C. amphora $\left(\mathrm{T}_{97,0.05}=4.85\right)$, C. crulsi $\left(\mathrm{T}_{161,0.05}=9.60\right)$ e $C$. manauara $\left(\mathrm{T}_{69,0.05}=4.99\right)$, todas com $\mathrm{P}<0.001$. Não houve diferença estatística no tamanho das aranhas entre reservas pequenas, grandes e bordas (ANOVA $\mathrm{P}>0.05$ para as quatro espécies). Também não foram detectadas diferenças do tamanho das fêmeas apenas entre as reservas pequenas e grandes (Mann-Withney, p>0.05) (figura 2). O número de réplicas impede comparações de tamanhos de aranhas entre cada uma das situações de tamanho de reserva e posição do transecto entre centro, borda e mata secundária.

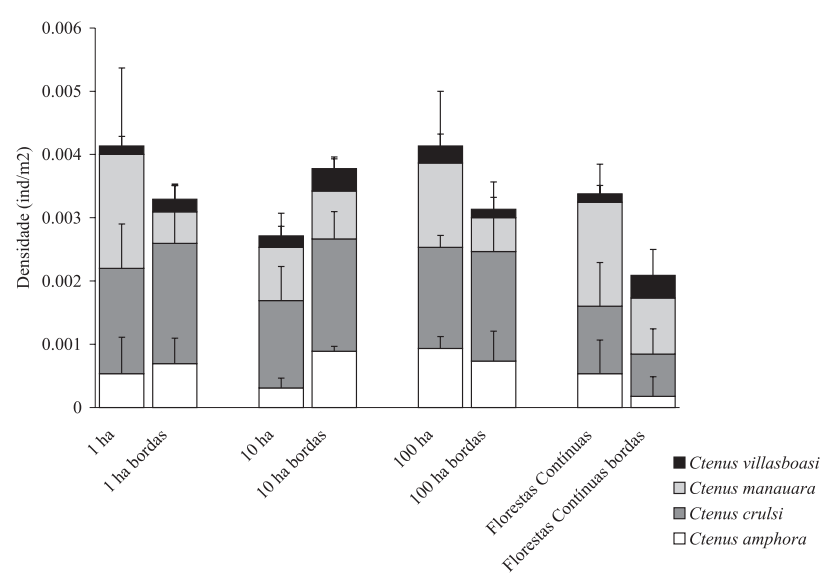

Figura 1 - Densidades e proporções das quatro espécies de aranhas Ctenus nas diferentes categorias de tamanho de fragmentos e em floresta contínua de terra-firme na Amazônia Central.

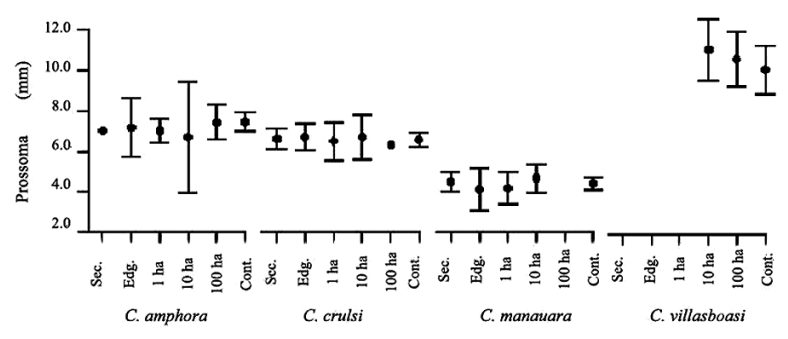

Figura 2 - Comprimento do prossoma de fêmeas adultas das quatro espécies de aranhas Ctenus nas categorias de tamanhos de reservas $(\mathrm{Sec} .=$ Florestas Secundárias, Edg. $=$ Bordas, Cont. $=$ Florestas Contínuas). As barras representam os desvios padrões das médias.

\section{DISCUSSÃO}

Os resultados do presente estudo não indicaram que existe um efeito de fragmentação ou efeito de borda sobre as quatro espécies de aranhas do gênero Ctenus. No entanto, em 2001 e 2002 os mesmos fragmentos florestais foram novamente amostrados por Rego et al. (2007) e foram constatadas que as densidades de duas espécies de Ctenus ( $C$. amphora e $C$. villasboasi) são significativamente menores nos fragmentos que na floresta contínua. Estes resultados desiguais podem ser atribuídos às diferenças nas épocas de amostragem dos dois estudos e as diferentes condiçôes dos fragmentos nestes diferentes períodos. O estudo de Rego et al. (2007) foi realizado depois da retirada da vegetação secundária das bordas de alguns dos fragmentos estudados.

O estudo de Rego et al. (2007) mostrou que a densidade de $C$. amphora e de $C$. villasboasi é significativamente maior na floresta continua que nos fragmentos, e que a densidade C. manauara e C. crulsi foram similares. Além disso, Rego et al. (2007) mostraram que a densidade de indivíduos no interior da floresta e nas suas bordas não foram significativas para nenhuma das espécies, sugerindo que a queda nas populaçôes de $C$. amphora e $C$. villasboasi nos fragmentos deve ter sido causada principalmente pelo isolamento da floresta. Estes resultados observados em conjunto aos do presente estudo reforçam a suposição de que o isolamento das reservas realmente influencia as populações de aranhas Ctenus nestas áreas, mas que o crescimento da floresta secundária ao redor das reservas parece restabelecer, para fatores importantes na ecologia destas aranhas, uma situação semelhante à do seu habitat original. Assim, é possível propor que o efeito da fragmentação florestal sobre as populaçôes de Ctenus no período de tempo do presente estudo deve ter sido diminuído quando a floresta ao redor dos fragmentos se regenerou e que a resposta deste grupo parece ser muito rápida.

Sugerimos que o estudo do efeito da fragmentação sobre estes artrópodes seja feito em fragmentos menores e que sejam feitos isolamentos das áreas em períodos mais curtos, isolando a influencia da floresta secundária. Devem-se também considerar outros fatores que podem influenciar a densidade e a distribuição das espécies de aranhas Ctenus, como sazonalidade (Gasnier, 1996), tipos de solo (Gasnier \& Höfer, 2001), grau de perturbação (Jocqué et al., 2005), presença de formigas de correição Eciton burchelli (Gasnier et al., 1995, Vieira \& Höfer 1994), competição interespecífica (Gasnier et al., 1995; Rego et al., 2005) e diferenças entre detectabilidade de machos e fêmeas (machos são mais ativos que as fêmeas, Salvestrini \& Gasnier, 2001; Gasnier et al., 2002).

Ainda é importante considerar algumas conclusões obtidas neste estudo que permitem uma melhor compreensão da ecologia das populaçôes de Ctenus em floresta de terra firme. As proporçôes entre as espécies estudadas foram semelhantes 
às encontradas nos estudos anteriores com estas aranhas sobre este tipo de solo (Höfer et al., 1994; Höfer \& Brescovit, 2001, Gasnier, 1996), sendo ordenadas decrescentemente na ordem C. crulsi, C. manauara, C. amphora e C. villasboasi. Ainda coincidindo com outros estudos realizados nas áreas observamos que o número de fêmeas adultas é superior ao de machos, sendo uma provável reflexão da diferença de mobilidade entre os sexos (Höfer et al., 1994; Gasnier, 1996). As variações da freqüência das quatro espécies em diferentes amostragens reforçam o sugerido por Gasnier (1996) de que estas espécies têm uma variação sazonal na abundância (menores abundâncias na época seca). Também é reforçada a conclusão de Gasnier (1996) e Gasnier et al. (2002) de que há um dimorfismo sexual em tamanho entre os sexos para estas espécies.

A relevância dos estudos que relacionam espécies dependentes da disponibilidade de insetos é evidente, pois a resposta sobre estas espécies indica indiretamente as condiçōes atuais de suas presas e da comunidade de invertebrados como um todo. A rápida recomposição da densidade de aranhas pode indicar que a reconstituição da fauna de invertebrados pelos quais estas se alimentam é também rápida, ou que ao menos existe uma adaptação destas populações a condições de ambientes fragmentados. Assim, novos estudos direcionados poderão esclarecer consideravelmente quais são os principais fatores que ajudam ou impedem a recomposição das populações afetadas pelo desmatamento e fragmentação florestal.

\section{AGRADECIMENTOS}

Agradecemos a Antonio Cardoso, Francisco M. Bezerra, João de Deus Farias, José F. Tenassol Jr., Luiz C. Prata, Osmaildo F. Silva pelo auxílio nos trabalhos de campo. Especialmente a Luiz Dario M. Mestre que proporcionou ao autor sênior condições de realizar este estudo. O suporte financeiro para as fases de campo foi cedido pelo Projeto Dinâmica Biológica de Fragmentos Florestais (INPA/ Smithsonian Tropical Research Institution) e Conselho Nacional de Desenvolvimento Científico e Tecnológico (CNPq-Brasil; Projeto 400023/98). Essa publicação recebeu o n 506 na série técnica do PDBFF.

\section{BIBLIOGRAFIA CITADA}

Almeida, C.E.; Ramos, E.F.; Gouvea, E.; Carmo-Silva, M.; Costa, J. 2000. Natural history of Ctenus medius Keyserling, 1891 (Araneae, Ctenidae): observations on habitats and the development of chromatic patterns. Brazilian Journal of Biology, 60(3): 503-509.

Baldissera R.; Ganade G.; Fontoura S.B. 2004. Web spider community response along an edge between pasture and Araucaria forest. Biological Conservation, 118:403-409.
Baz, A.; Garcia-Boyero, A. 1995. The Effects of Forest Fragmentation on Butterfly Communities in Central Spain. Journal of Biogeography, 22(1): 129-140.

Bierregaard, R.O. Jr.; Lovejoy, T.E., Kapos, V.; Santos, A.A.; Hutchings, R.W. 1992. The biological Dynamics of Tropical Forest Fragments. Bioscience, 42(11): 859-66.

Bolger, D.T.; Suarez, A.V.; Crooks, K.R.; Morrison, S.A.; Case, T.J. 2000. Arthropods in urban habitat fragmentation in southern California: area, age, and edge effects. Ecological Applications, 10(4): 1230-1248.

Brown, K.S. Jr.; Hutchings, R. 1997. Disturbance, Fragmentation, and the Dynamics of diversity in Amazoninan Forest Butterflies. In: Laurance, W. F., Bierregaard, R. O. (Eds.). Tropical Forest Remnants: Ecology, Management, and Conservation of Fragmented Communities. University of Chicago Press, Chicago. p. 91110.

Didham, K.R.; Ghazoul J.; Stork, N.E.; Davis, A. 1996. Insects in fragmented forest: a functional approach. Tree, 11 (6): 255260.

Didham, K.R; Lawton, J.H.; Hammond, P.M.; Eggleton, P. 1998. Trophic structure, stability and extintion dynamics of beetles (Coleoptera) in tropical forest fragments. Phil. Trans. R. Soc. Lond., 353: 437-451.

Folly-Ramos, E.; Almeida, C.E.; Carmo-Silva, M.; Costa J. 2002. Natural history of Ctenus medius Keyserling, 1891 (Aranae, Ctenidae) II: life cycle and aspects of reproductive behavior under laboratory conditions. Brazilian Journal of Biology, 62 (4b): 87-793.

Gasnier, T.R.; Höfer, H.; Brescovit, A. D. 1995. Factors affecting the "activity density" of spiders on tree trunks in Amazonian Rainforest. Ecotropica, 1 (2): 69-77.

Gasnier, T.R. 1996. Ecologia comparada de quatro espécies de aranhas errantes do gênero Ctenus (Walkenaer) (Araneae, Ctenidae) em uma floresta na Amazônia Central: Bases para um modelo integrado de coexistência. Tese de Doutorado. Instituto Nacional de Pesquisas da Amazônia (INPA), Fundação Universidade do Amazonas. 47pp.

Gasnier, T.R.; Höfer, H. 2001. Patterns of abundance of four species of wandering spiders (Ctenidae, Ctenus) in a forest in central Amazonia. Journal of Arachnology, 29: 95-103.

Gasnier, T.R.; Azevedo, C.S.; Torres-Sanchez, M.P.; Höfer, H. 2002. Adult size of eight hunting spider species in Central Amazonia: temporal variations and sexual dimorphisms. Journal of Arachnology, 30: 149-154.

Gasnier, T.R.; Höfer, H.; Torres-Sanchez, M.P.; Azevedo, C. (no prelo). História natural de algumas espécies de aranhas das famílias Ctenidae, Pisauridae e Lycosidae na Reserva Ducke: bases para um modelo integrado de coexistência. In: Fonseca, C.R. V.; Magalhães, C.; Rafael, J.A.; Franklin, E. (Eds.). A Fauna de Artrópodes da Reserva Florestal Adolpho Ducke, Estado Atual do Conhecimento Taxonômico e Biológico.

Hagan, J.M.; Haegen, W.M.V; Mckinley, P. 1996. The early development of forest fragmentation effects on birds. Conservation Biology, 10 (1): 188-202. 
Harris, L.D. 1988. Edge effects and conservation of biotic diversity. Conservation Biology, 2: 330-332.

Höfer, H.; Brescovit, A. D.; Gasnier, T.R.H. 1994. The wandering spiders of the genus Ctenus (Ctenidae, Araneae) of Reserva Ducke, a rainforest reserve on central Amazonia. Andrias, 13:81-98.

Höfer, H.; Brescovit, A.D. 2001. Species and guild struture of Neotropical spider assemblage (Araneae) from Reserva Ducke, Amazonas, Brasil. Andrias, 15: 99-119.

Jocqué, R. 1981a. On reduced size in spiders from marginal habitats. Oecologia, 49: 404-408.

Jocqué, R. 1981b. Size and weight variations in spiders and their ecological significance: Biologish Jaarboek Dodonaea, 49: 155-165.

Jocqué, R.; Samu, F; Bird, T. 2005. Density of spiders (Araneae: Ctenidae) in Ivory Coast rainforests. J. Zool. (Lond.), 266:105110.

Klein, B.C. 1989. Effects of forest fragmentation on dung and carrion beetle communities in central Amazonia. Ecology, 70: $1715-1725$.

Lovejoy, T.E. 1980. Discontinous wilderness: minimum areas for conservation. Parks, 5 (2): 13-15.

Lovejoy, T.E.; Bierregard, R.O.; Rankin, J.M.; Schubart, H.O.R. 1983. Ecological dynamics of forest fragments. In: Sutton, S.L.; Witmore, T.C.; Chadwick, A.C. (Eds.). Tropical Rainforest: Ecology and Management. Blackwell, Scient. Publ. Oxford. p. 377-385.

Lovejoy, T.E.; Bierregaard, R.O.; Rylands, A.B.; Malcom, J.R.; Quintela, C.E.; Harper, L.H.; Brown K.S Powell; A.H., Powell, G.V.N.; Schubart, H.O.R.; Hays M.S. 1986. Edge and other effects of isolation on Amazon forest fragments. In: Soulé M.E. (Ed.). Conservation Biology: The science of scarcity and diversity. Sinauer Associates, Sunderland, M.A. p. 257-285.

Marques-Filho, A.O.; Ribeiro, M.N.G.; Santos, H.M.; Santos, J.M. 1981. Estudos climatológicos da Reserva Florestal Ducke. IV. Precipitação. Acta Amazônica, 11:759-768.

Miyashita, T.; Shinkai, A.; Chida, T. 1998. The effects of forest fragmentation on web spider communities in urban areas. Biological Conservation, 86: (3) 357-364.

Offerman, H.L.; Dale, V.H.; Pearson, S.M.; Bierregaard Jr., R.O.; O'Neill, R. V. 1995. Effects of forest fragmentation on neotropical fauna: current research and data availability. Environ. Rev. 3: 191-211.
Rego, F.N.N.A.; Venticinque, E.M.; Brescovit, A.D. 2005. Densidades de aranhas errantes (Ctenidae e Sparassidae: Araneae) em uma floresta fragmentada. Biota Neotropica, Número Especial 5: (1a). http://www.biotaneotropica.org.br/v5n1a/pt/ abstract?article+BN002051a2005. ISSN 1676-0603.

Rego, F.N.A.; Venticinque, E.M.; Brescovit, A.D. 2007. Effects of forest fragmentation on four Ctenus spider populations (Araneae, Ctenidae) in central Amazonia, Brazil. Studies on Neotropical Fauna and Environment. no prelo.

Salati, E.; Ribeiro, M.N.G.; Absy, M.L.; Nelson, B.W. 1991. Clima da Amazônia: Presente, Passado e Futuro. Pp. 21-34, In: Val, A.L.; Figliuolo, R.; Feldberg, E. (Eds.). Bases Cientificas para Estratégias de Preservação e Desenvolvimento da Amazônia: Fatos e Perspectivas, V 1. Instituto Nacional de Pesquisas da Amazônia, Manaus.

Souza, O.F.F.; Bronw, V.K. 1994. Effects of habitat fragmentation on Amazonian termite communities. Journal of Tropical Ecology, 10: 197-206.

Stouffer, P.C.; Bierregaard Jr., R.O. 1995. Use of amazonian forest fragments by understory insectivorous birds. Ecology, 76 (8): 2429-2445.

Salvestrini, F.M.D.; Gasnier, T.R. 2001. Differeneces in the activity of juveniles, females and males of two hunting spiders of the genus Ctenus (Araneae, Ctenidae): active males or inactive females? Journal of Arachnology, 29: 276-278.

Vasconcelos, H.L.; Carvalho, K.S.; Delabie, J.H.C. 2001. Landscape modifications and ant communities. In: Bierregaard Jr, R.O.; Gascon, C.; Lovejoy, T.E.; Mesquita, R. (Eds.). Lessons From Amazonia: The Ecology and Conservation of a Fragmented Forest. Yale University Press, New Haven. p. 199-206.

Venticinque, E.M.; Fowler, H.G. 2001. Local Extinction Risks and Asynchronies: The evidence for a Metapopulation Dynamics of a Social Spider, Anelosimus eximius (Araneae, Theridiidae). In: Bierregaard Jr., R.O.; Gascon, C.; Lovejoy, T.E.; Mesquita, R. (Eds.). Lessons From Amazonia: The Ecology and Conservation of a Fragmented Forest. Yale University Press, New Haven. p. 187-198.

Vieira, R.S.; Höfer, H. 1994. Prey spectrum of two army ant species in central Amazonia, with special attention on their effect on spider populations. Andrias, 13:189-198.

Recebido em 14/12/2005

Aceito em 19/03/2007 